Article

\title{
Pangenotypic and Genotype-Specific Antivirals in the Treatment of HCV Genotype 4 Infected Patients with HCV Monoinfection and HIV/HCV Coinfection
}

\author{
Dorota Zarębska-Michaluk 1,*(D), Jerzy Jaroszewicz ${ }^{2}$, Anna Parfieniuk-Kowerda ${ }^{3}$, Małgorzata Pawłowska ${ }^{4}$ (D), \\ Ewa Janczewska ${ }^{5}$, Hanna Berak ${ }^{6}$, Justyna Janocha-Litwin ${ }^{7}$, Jakub Klapaczyński ${ }^{8}$, Krzysztof Tomasiewicz ${ }^{9}$, \\ Anna Piekarska ${ }^{10}$, Rafał Krygier ${ }^{11}$ (D), Jolanta Citko ${ }^{12}$, Olga Tronina ${ }^{13}$, Krystyna Dobrowolska ${ }^{14}$ \\ and Robert Flisiak ${ }^{3, *}$ (D)
}

check for

updates

Citation: Zarębska-Michaluk, D.; Jaroszewicz, J.; Parfieniuk-Kowerda, A.; Pawłowska, M.; Janczewska, E.; Berak, H.; Janocha-Litwin, J.; Klapaczyński, J.; Tomasiewicz, K.; Piekarska, A.; et al. Pangenotypic and Genotype-Specific Antivirals in the Treatment of HCV Genotype 4 Infected Patients with HCV Monoinfection and $\mathrm{HIV} / \mathrm{HCV}$ Coinfection. J. Clin. Med. 2022, 11 389. https://doi.org/10.3390/ jcm11020389

Academic Editors: Giovanni Tarantino and Hiroki Nishikawa

Received: 28 November 2021 Accepted: 11 January 2022 Published: 13 January 2022

Publisher's Note: MDPI stays neutral with regard to jurisdictional claims in published maps and institutional affiliations.

Copyright: (C) 2022 by the authors. Licensee MDPI, Basel, Switzerland. This article is an open access article distributed under the terms and conditions of the Creative Commons Attribution (CC BY) license (https:// creativecommons.org/licenses/by/ $4.0 /)$.
1 Department of Infectious Diseases, Jan Kochanowski University Kielce, 25-516 Kielce, Poland

2 Department of Infectious Diseases and Hepatology, Medical University of Silesia in Katowice, 40-055 Katowice, Poland; jerzy.jr@gmail.com

3 Department of Infectious Diseases and Hepatology, Medical University of Białystok, 15-540 Białystok, Poland; anna.parfieniuk@gmail.com

4 Department of Infectious Diseases and Hepatology, Ludwik Rydygier Collegium Medicum, Bydgoszcz Faculty of Medicine Nicolaus Copernicus University in Torun, 85-030 Bydgoszcz, Poland; mpawlowska@cm.umk.pl

5 Faculty of Health Sciences in Bytom, Department of Basic Medical Sciences, Medical University of Silesia, ID Clinic, Hepatology Outpatient Department, 41-902 Bytom, Poland; e.janczewska@poczta.fm

6 Hospital for Infectious Diseases in Warszawa, 02-091 Warszawa, Poland; hberak@wp.pl

7 Department of Infectious Diseases and Hepatology, Medical University Wrocław, 50-367 Wrocław, Poland; justynajanocha@o2.pl

8 Department of Internal Medicine and Hepatology, Central Clinical Hospital of the Ministry of Internal Affairs and Administration, 02-507 Warszawa, Poland; klapaj@gmail.com

9 Department of Infectious Diseases, Medical University of Lublin, 20-059 Lublin, Poland; tomaskdr@poczta.fm

10 Department of Infectious Diseases and Hepatology, Medical University of Łódź, 90-419 Łódź, Poland; annapiekar@gmail.com

11 Outpatients Hepatology Department, State University of Applied Sciences, 62-510 Konin, Poland; rafalkrygier@gmail.com

12 Medical Practice of Infections, Regional Hospital, 10-561 Olsztyn, Poland; citkoj@wss.olsztyn.pl

13 Department of Transplantation Medicine, Nephrology, and Internal Diseases, Medical University of Warsaw, 02-006 Warszawa, Poland; olgatronina@wp.pl

14 Collegium Medicum, Jan Kochanowski University, 25-516 Kielce, Poland; krystyna.dobrowolska98@gmail.com

* Correspondence: dorota1010@tlen.pl (D.Z.-M.); robert.flisiak1@gmail.com (R.F.)

\begin{abstract}
The introduction of the direct-acting antivirals (DAA) has substantially improved the effectiveness of the therapy in patients with chronic hepatitis C. We aimed to compare the efficacy of pangenotypic and genotype-specific DAA in the cohort of genotype (GT) four patients with HCV monoinfection and HIV coinfection. A total of 662 GT4-infected patients treated in 2015-2020-of whom 168 (25.3\%) were coinfected with HIV, selected from the retrospective EpiTer-2 database-were enrolled in the analysis. Among HIV-coinfected patients, 54\% (90) were treated with genotypespecific regimens and $46 \%$ (78) with pangenotypic options, while among HCV-monoinfected patients, the rates were $72 \%$ and $28 \%$, respectively. Significantly higher rate of males $(67.9 \%$ vs. $57.7 \%$, $p=0.01)$, a lower rate of liver cirrhosis $(10.2 \%$ vs. $18.1 \%, p=0.02)$, and higher of treatment-naïve patients $(87.5 \%$ vs. $76.7 \%, p=0.003)$ were documented in the HIV coinfected population. The overall sustained virologic response after exclusion of non-virologic failures was achieved in $98 \%$ with no significant difference between HIV-positive and HIV-negative patients, $96.2 \%$ vs. $98.5 \%$, respectively. While the genotype-specific regimens resulted in a similar cure rate regardless of the HIV status, the pangenotypic options were more efficacious in patients with HCV monoinfection $(99.3 \%$ vs. $94.4 \%, p=0.05)$. Hereby, we demonstrated the high effectiveness and good safety profile of the DAA therapy in the population of HCV GT4 infected patients with HIV coinfection supporting the
\end{abstract}


current recommendations to treat $\mathrm{HCV} / \mathrm{HIV}$ coinfected patients with the same options as those with HCV monoinfection.

Keywords: hepatitis C virus; genotype 4; human immunodeficiency virus; direct-acting antivirals; pangenotypic; genotype-specific

\section{Introduction}

The hepatitis $\mathrm{C}$ virus (HCV)—belonging to the genus Hepacivirus of the family Flaviviridae-is a small, enveloped, single-stranded, positive-sense ribonucleic acid (RNA) virus. Due to the wide genetic diversity of the viral genome, six major HCV genotypes (GT) with more than $30 \%$ difference in RNA sequence were described [1].

The most prevalent worldwide is GT1, accounting for nearly $50 \%$ of all cases of $\mathrm{HCV}$, followed by the GT3 responsible for 30\% of infections; while GT4 with an overall rate of $8 \%$ is the third by frequency [1]. The GT1 and GT3 infections dominate in most countries globally irrespective of the economic status, whereas the GT4 is more common in lowincome countries. The highest prevalence of GT4 is documented in Sub-Saharan and North Africa and the Middle East [1]. The frequency of GT4 infections in Europe varies across countries; however, a growing prevalence has been observed in recent years in the south of the continent in the Mediterranean Sea region due to immigration [2,3]. An increasing share of GT4 has also been documented in Europe in people who inject drugs and patients coinfected with human immunodeficiency virus (HIV) [2,4,5].

In the interferon (IFN) era, the effectiveness of the antiviral therapy with pegylated IFN (pegIFN) and ribavirin (RBV) in GT4 was higher than achieved in the GT1 infected patients and lower compared to those with GT2 and GT3 infections [6]. The introduction of the firstclass protease inhibitors, telaprevir (TVR) and boceprevir (BOC), used in combination with pegIFN and RBV has turned the situation into a disadvantage since these new drugs were active only in GT1-infected patients [7]. The next generation of direct-acting antiviralsprotease inhibitor simeprevir (SMV), polymerase inhibitor sofosbuvir (SOF), and inhibitor of non-structural protein (NS) 5A daclatasvir (DCV)-were registered to use with the combination of pegIFN and RBV in GT4 infected patients with significant improvement in sustained virologic response (SVR) [8-10].

However, IFN-containing therapy in patients with coinfection with human immunodeficiency virus (HIV) was more difficult compared to those with HCV monoinfection. Difficulties in therapy resulted not only from lower response rates, but also from barriers in treatment use including the comorbidities, limited life expectancy, unfavorable safety profile, and non-adherence to therapy [11].

The real breakthrough in the treatment of GT4 infection was the implementation of direct-acting antivirals (DAA) [12]. The higher efficacy, substantially better tolerability, and shorter treatment duration broke down limitations associated with HIVcoinfection and has enabled wider access to antiviral therapy and eradicated HCV in this subpopulation. Genotype-specific regimens for GT4-infected patients, including ombitasvir/paritaprevir/ritonavir (OPr) + RBV, sofosbuvir/ledipasvir (SOF/LDV) $\pm \mathrm{RBV}$, and grazoprevir/elbasvir (GZR/ELB) $\pm \mathrm{RBV}$ resulted in high treatment efficacy. Therefore, the first available pangenotypic options-SOF + RBV and SOF + DCV $\pm \mathrm{RBV}$-were infrequently used in this population [13]. The application of pangenotypic regimens in GT4 patients has increased with the advent of new drug formulations, glecaprevir/pibrentasvir (GLE/PIB) and sofosbuvir/velpatasvir (SOF/VEL) \pm RBV. Genotype-specific regimens have been available in Poland since mid-2015, pangenotypic therapies have been available since mid-2018 and there have been no restrictions on access to therapy for HIV coinfected patients.

Clinical trials on DAA regimens evaluating efficacy and safety in HCV / HIV coinfected population have demonstrated high cure rates and good tolerablity relative to IFN-based 
therapies; however, data on the real-world effectiveness of DAA, especially pangenotypic regimens, in HIV-positive patients with GT4 infection are limited [14-18]. Therefore, the current analysis aimed to evaluate the IFN-free treatment in the cohort of GT4 patients with HIV coinfection considering the type of therapy used.

\section{Materials and Methods}

\subsection{Study Design and Materials}

The patients included in the current analysis were selected from the EpiTer-2 database (of 13,552 patients treated for chronic hepatitis C in 2015-2020 (Figure 1), (http:/ / www pteilchz.org.pl/informacje/epiter-2/ assessed on 28 December 2021). This is an ongoing large retrospective multicenter national real-world study evaluating DAA-based antiviral treatment in individuals with chronic hepatitis $C$ treated in 22 Polish hepatology centers. The selection of the antiviral regimen was made by the treating physicians based on the medical knowledge according to the national recommendations of the Polish Group of Experts for HCV and the principles of the reimbursed therapeutic program established by the National Health Fund (NHF) considering the potential drug-drug interactions with antiretroviral therapy (ART) [19]. Drug doses and treatment duration were consistent with the Summary of Product Characteristics.

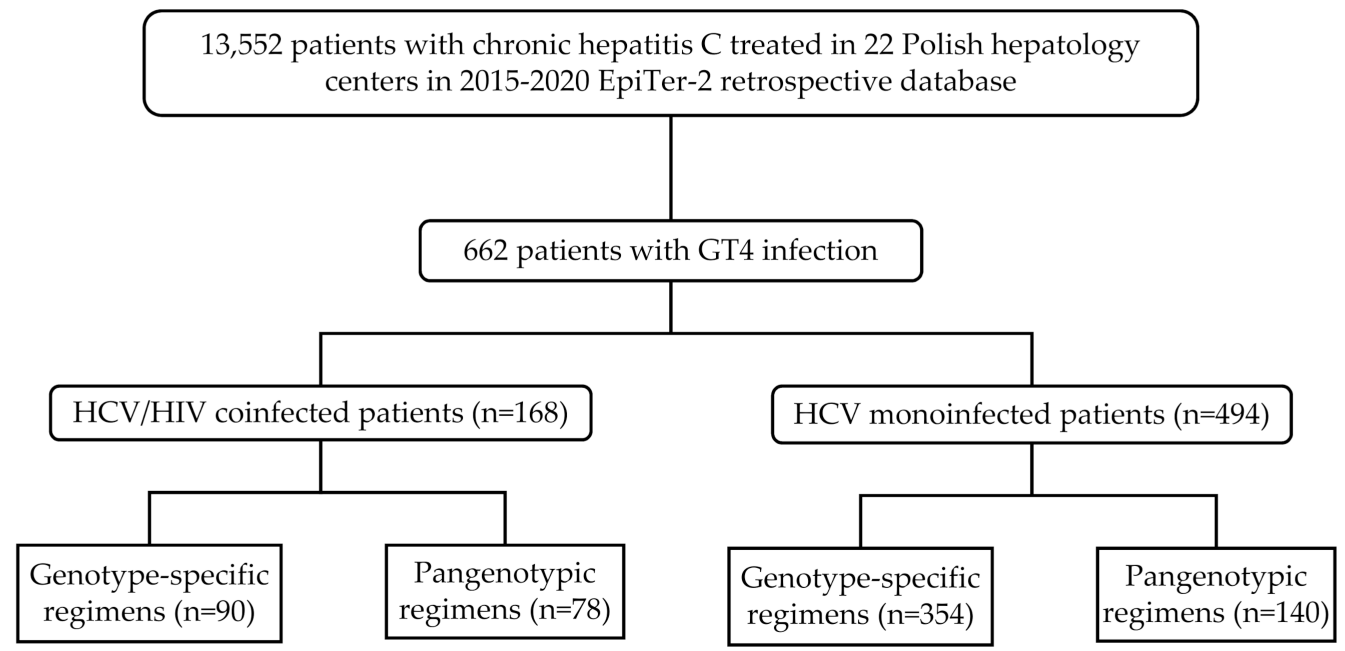

Figure 1. Flow chart showing the selection and stratification of patients included in the study.

Patients provided informed consent before the start of the treatment according to the requirements of NHF. Patients' data were collected retrospectively and submitted via a web platform operated by Tiba sp. z o.o. following the National General Data Protection Regulation in Poland.

The information captured at baseline included demographic and clinical data: age, gender, body mass index (BMI), comorbidities and concomitant medications, the severity of liver disease, hepatitis B virus (HBV) and human immunodeficiency virus (HIV) coinfections, and the history of previous antiviral treatment.

The degree of liver disease was evaluated noninvasively by the transient elastography (TE) or shear-wave elastography (SWE), or histologically by the liver biopsy. The measurement of the liver stiffness was a basis for assigning the patient to the fibrosis stage F0-F4 according to the METAVIR score using the recommendations of the European Association for the Study of the Liver (EASL) with 13 kilopascals as a threshold to define liver cirrhosis [20]. The cirrhotic patients were evaluated for the presence of esophageal varices and scored in the Child-Pugh (CP) scale and Model of End-Stage Liver Disease (MELD).

Characteristics of the patients at the start of the therapy also included laboratory data such as the activity of the serum alanine transaminase (ALT); the concentration of the 
bilirubin, albumin, creatinine, and hemoglobin; white blood cell and platelet counts; and $\mathrm{HCV}$ viral load.

The HCV RNA was assessed at baseline, at the end of treatment, and at least 12 weeks after therapy completion.

The assessment of the viral load was performed by the real-time polymerase chain reaction assays and genotyping was performed using reverse hybridization assays.

\subsection{Efficacy and Safety Evaluation}

The primary efficacy outcome was the sustained virologic response (SVR) defined as the negative result of HCV RNA 12 weeks after treatment. The secondary outcome was the effectiveness of the therapy depending on the HIV status and type of regimen-genotypespecific versus pangenotypic.

Genotype-specific regimens include OPr + RBV, GZR/EBR, and SOF/LDV, while the pangenotypic regimens were as follows: $\mathrm{SOF} \pm \mathrm{DCV} \pm \mathrm{RBV}$, SOF/VEL $\pm \mathrm{RBV}$, and GLE/PIB.

The safety of the therapy was assessed by the rate of the treatment course modification and discontinuation, the prevalence of adverse events (AE) including the severe $\mathrm{AE}$, and deaths. The AE of particular interest associated with the deterioration of the liver function such as ascites, encephalopathy, and gastrointestinal bleeding were assessed in patients with liver cirrhosis.

The intention-to-treat group (ITT) included patients who received at least one dose of antiviral drug, and the per-protocol group (PP) was established by excluding patients because of non-virologic failure.

\subsection{Statistical Analysis}

Results were expressed as mean \pm standard deviation (SD) and median with $10-90 \%$ confidence intervals or number (percentage). A $p$-value of less than 0.05 was considered significant. The significance of differences was calculated by the $\chi 2$ or Fisher exact tests for nominal variables and by the Mann-Whitney test and the Kruskal-Wallis analysis of variance for continuous variables. For outcome analyses, odds ratios with $95 \%$ confidence intervals were additionally calculated. Univariable comparisons were calculated using the GraphPad Prism 5.1 software (GraphPad Software, Inc., La Jolla, CA, USA).

\section{Results}

\subsection{Characteristics of the Study Population}

A total of 662 patients with HCV GT4 infection with mean age $45.3 \pm 12.6$ years, male predominance $(60.3 \%)$, and $15.9 \%$ rate of liver cirrhosis treated in $2015-2020$ were enrolled in the analysis (Figure 1). Most of them were treatment-naïve (79.5\%) and the most commonly used DAA regimen in the current therapy was OPr + RBV (39.6\%). One hundred sixty-eight patients (25.3\%) were coinfected with HIV and 162 of them $(96.4 \%)$ were on the antiretroviral therapy with the most common regimen $(121 ; 74.7 \%)$ consisted of emtricitabine and tenofovir alafenamide (109 patients) or disoproxil (12 patients) followed by the abacavir and lamivudine-based option (22; 13.6\%). In the remaining 19 patients, other therapeutic options were used.

A significantly higher rate of males $(67.9 \%$ vs. $57.7 \%, p=0.01)$ and a higher percentage of concomitant medications ( $97.6 \%$ vs. $52.2 \%, p<0.001$ ) were documented in $\mathrm{HCV} / \mathrm{HIV}$ coinfected compared to patients with HCV monoinfection, while monoinfected individuals were more frequently diagnosed with comorbidities $(59.5 \%$ vs. $38.1 \%, p<0.001)$, the statistically significant difference was found for arterial hypertension and kidney diseases $(p<0.001)$ (Table 1$)$. 
Table 1. Baseline characteristics of GT4-infected patients depending on the HIV status.

\begin{tabular}{|c|c|c|c|}
\hline Parameter & $\begin{array}{c}\text { HIV+ } \\
n=168\end{array}$ & $\begin{array}{l}\text { HIV- } \\
\mathrm{n}=494\end{array}$ & $p=$ \\
\hline Gender, females/males, n (\%) & $54(32.1) / 114(67.9)$ & $209(42.2) / 285(57.7)$ & 0.01 \\
\hline Age (years) mean (SD) & $43.3(7.7)$ & $45.9(13.8)$ & \multirow{2}{*}{0.11} \\
\hline Median $(10-90 \% C I)$ & $43(34-53)$ & $44(28-64)$ & \\
\hline BMI mean (SD) & $25.2(3.9)$ & $26.0(4.6)$ & \multirow[b]{2}{*}{0.19} \\
\hline Median $(10-90 \% C I)$ & $25.3(20.2-30.3)$ & $25.3(20.6-32.4)$ & \\
\hline Any comorbidity, n (\%) & $64(38.1)$ & $294(59.5)$ & $<0.001$ \\
\hline Arterial hypertension & $15(8.9)$ & $149(30.2)$ & $<0.001$ \\
\hline Diabetes & $6(3.6)$ & $40(8.1)$ & 0.05 \\
\hline Autoimmune diseases & $1(0.6)$ & $15(3)$ & 0.08 \\
\hline Kidney diseases & $2(1.2)$ & $48(9.7)$ & $<0.001$ \\
\hline incl. kidney transplantation & 0 & $29(5.9)$ & $<0.001$ \\
\hline Non-HCC tumors & $1(0.6)$ & $7(1.4)$ & 0.67 \\
\hline Depression & $8(4.8)$ & $25(5.1)$ & 1 \\
\hline Other & $52(31)$ & $184(37.2)$ & 0.16 \\
\hline Concomitant medications, $\mathrm{n}(\%)$ & $164(97.6)$ & $258(52.2)$ & $<0.001$ \\
\hline ALT IU/L, mean (SD) & $70(94)$ & $71(59)$ & \multirow{2}{*}{0.01} \\
\hline Median $(10-90 \% \mathrm{CI})$ & $45(22-135)$ & $53(22-140)$ & \\
\hline Bilirubin mg/dL, mean (SD) & $0.7(0.5)$ & $0.7(0.5)$ & \multirow{2}{*}{0.02} \\
\hline Median $(10-90 \% C I)$ & $0.5(0.2-1.1)$ & $0.6(0.3-1.4)$ & \\
\hline Albumin g/dL, mean (SD) & $4.1(0.5)$ & $4.1(0.5)$ & \multirow{2}{*}{0.36} \\
\hline Median $(10-90 \% \mathrm{CI})$ & $4.1(3.5-4.6)$ & $4.1(3.5-4.7)$ & \\
\hline Creatinine $\mathrm{mg} / \mathrm{dL}$, mean (SD) & $0.9(0.2)$ & $1.1(1.2)$ & \multirow{2}{*}{0.01} \\
\hline Median $(10-90 \% \mathrm{CI})$ & $0.9(0.6-1.1)$ & $0.8(0.6-1.2)$ & \\
\hline Hemoglobin g/dL, mean (SD) & $14.7(1.7)$ & $14.5(1.8)$ & \multirow{2}{*}{0.4} \\
\hline Median $(10-90 \% \mathrm{CI})$ & $14.7(12.5-16.9)$ & $14.7(12.2-16.6)$ & \\
\hline Platelets, $\times 1000 / \mu \mathrm{L}$, mean (SD) & $210(86)$ & $200(71)$ & \multirow{2}{*}{0.4} \\
\hline Median $(10-90 \% \mathrm{CI})$ & $209(123-284)$ & $201(101-286)$ & \\
\hline $\mathrm{HCV}$ RNA $\times 10^{6} \mathrm{IU} / \mathrm{mL}$, mean $(\mathrm{SD})$ & $2.4(3.4)$ & $2.72(9.55)$ & \multirow{2}{*}{0.45} \\
\hline Median $(10-90 \% \mathrm{CI})$ & $0.9(0.05-7.8)$ & $1.1(0.1-5.6)$ & \\
\hline
\end{tabular}

GT, genotype; HIV, human immunodeficiency virus; SD, standard deviation; BMI, body mass index; non-HCC non-hepatocellular carcinoma; ALT, alanine transaminase; HCV RNA, ribonucleic acid of hepatitis C virus.

The rate of patients with liver cirrhosis was significantly higher in the HCV monoinfected population ( $18.1 \%$ vs. $10.2 \%)$. The detailed characteristics regarding the severity of liver disease and HBV coinfection depending on the HIV status are presented in Table 2.

Table 2. Characteristics of the liver disease in GT4-infected patients depending on the HIV status.

\begin{tabular}{cccc}
\hline Parameter & $\begin{array}{c}\text { HIV+ } \\
\mathbf{n = 1 6 8}\end{array}$ & $\begin{array}{c}\text { HIV- } \\
\mathbf{n = 4 9 4}\end{array}$ & $p=$ \\
\hline Liver fibrosis, $\mathrm{n}(\%)$ & & & \\
F0 & $4(2.4)$ & $9(1.8)$ & \\
F1 & $88(52.4)$ & $241(48.9)$ & \\
F2 & $39(23.2)$ & $92(18.6)$ & 0.23 \\
F3 & $18(10.7)$ & $55(11.1)$ & \\
F4 & $17(10.1)$ & $88(17.8)$ & \\
no data & $2(1.2)$ & $9(1.8)$ & \\
F0-F3 & $149(89.8)$ & $397(81.9)$ & 0.02 \\
F4 & $17(10.2)$ & $88(18.1)$ & \\
\hline
\end{tabular}


Table 2. Cont.

\begin{tabular}{|c|c|c|c|}
\hline Parameter & $\begin{array}{c}\text { HIV+ } \\
n=168\end{array}$ & $\begin{array}{l}\text { HIV- } \\
\mathrm{n}=494\end{array}$ & $p=$ \\
\hline History of hepatic decompensation, $\mathrm{n}(\%)$ & $1(0.6)$ & $7(1.4)$ & \multirow{4}{*}{0.69} \\
\hline Ascites & 0 & $6(1.2)$ & \\
\hline Ascites+encephalopathy & $1(0.6)$ & 0 & \\
\hline Encephalopathy & 0 & $1(0.2)$ & \\
\hline Documented esophageal varices, n (\%) & $1(0.6)$ & $29(5.9)$ & 0.002 \\
\hline Hepatic decompensation at baseline, $\mathrm{n}(\%)$ & 0 & $5(1)$ & \multirow{3}{*}{0.33} \\
\hline Ascites & 0 & $3(0.6)$ & \\
\hline Encephalopathy & 0 & $2(0.4)$ & \\
\hline HCC history, n (\%) & 0 & $5(1)$ & 0.33 \\
\hline OLTx history, n (\%) & 0 & $3(0.6)$ & 0.57 \\
\hline \multicolumn{4}{|l|}{ Child-Pugh, n (\%) } \\
\hline B & $1(0.6)$ & $12(2.4)$ & 0.2 \\
\hline $\mathrm{C}$ & 0 & 0 & \\
\hline HBV coinfection (HBsAg+), n (\%) & $3(1.8)$ & $7(1.4)$ & 0.72 \\
\hline
\end{tabular}

GT, genotype; HIV, human immunodeficiency virus; HCC, hepatocellular carcinoma; OLTx, orthotopic liver transplantation; HBV, hepatitis B virus; HBsAg, hepatitis B surface antigen.

The higher rate of treatment-naïve patients (87.5\% vs. $76.7 \%)$ was demonstrated in HIV-positive compared to HIV-negative individuals (Table 3).

Table 3. Comparison of characteristics in GT4-infected patients depending on the HIV status.

\begin{tabular}{|c|c|c|c|}
\hline Parameter & $\begin{array}{c}\text { HIV+ } \\
n=168\end{array}$ & $\begin{array}{l}\text { HIV- } \\
\mathrm{n}=494\end{array}$ & $p=$ \\
\hline \multicolumn{4}{|l|}{ History of previous therapy, n (\%) } \\
\hline Treatment-naïve & $147(87.5)$ & $379(76.7)$ & \multirow{5}{*}{0.03} \\
\hline Nonresponder & $9(5.3)$ & $58(11.8)$ & \\
\hline Relapser & $6(3.6)$ & $20(4)$ & \\
\hline Discontinuation due to safety reason & $4(2.4)$ & $15(3)$ & \\
\hline Unknown type of response & $2(1.2)$ & $22(4.5)$ & \\
\hline Treatment-naïve & $147(87.5)$ & $379(76.7)$ & \multirow{2}{*}{0.003} \\
\hline Treatment-experienced & $21(12.5)$ & $115(23.3)$ & \\
\hline Previous regimen in treatment-experienced, $\mathrm{n}(\%)$ & $\mathrm{n}=21$ & $\mathrm{n}=115$ & \\
\hline IFN & 0 & $6(5.2)$ & 0.59 \\
\hline PegIFN + RBV & $15(71.4)$ & $93(80.8)$ & 0.38 \\
\hline $\mathrm{SOF}+$ PegIFN + RBV & 0 & $1(0.9)$ & 1 \\
\hline $\mathrm{SMV}+\mathrm{PegIFN}+\mathrm{RBV}$ & 0 & $6(5.2)$ & 0.59 \\
\hline $\mathrm{SOF}+\mathrm{RBV}$ & $1(4.8)$ & 0 & 0.15 \\
\hline $\mathrm{SOF} / \mathrm{LDV} \pm \mathrm{RBV}$ & 0 & $3(2.6)$ & 1 \\
\hline $\mathrm{OPr}+\mathrm{RBV}$ & $1(4.8)$ & $3(2.6)$ & 0.49 \\
\hline GZR/EBR & $1(4.8)$ & $1(0.9)$ & 0.29 \\
\hline $\mathrm{ASV}+\mathrm{DCV}$ & 0 & $1(0.9)$ & 1 \\
\hline No data & $3(14.2)$ & $1(0.9)$ & 0.01 \\
\hline Previous IFN-free regimen in patients with & $\mathrm{n}=21$ & $\mathrm{n}=115$ & \\
\hline treatment failure, $\mathrm{n}(\%)$ & $3(17.8)$ & $8(6.9)$ & 0.37 \\
\hline \multicolumn{4}{|l|}{ Current treatment regimens, $\mathrm{n}(\%)$} \\
\hline \multicolumn{4}{|l|}{ Genotype-specific } \\
\hline $\mathrm{OPr}+\mathrm{RBV}$ & $50(29.8)$ & $212(42.9)$ & 0.003 \\
\hline $\mathrm{GZR} / \mathrm{EBR} \pm \mathrm{RBV}$ & $38(22.6)$ & $124(25.1)$ & 0.53 \\
\hline $\mathrm{SOF} / \mathrm{LDV} \pm \mathrm{RBV}$ & $2(1.2)$ & $18(3.6)$ & 0.12 \\
\hline
\end{tabular}


Table 3. Cont.

\begin{tabular}{cccc}
\hline Parameter & $\begin{array}{c}\text { HIV+ } \\
\mathbf{n = 1 6 8}\end{array}$ & $\begin{array}{c}\text { HIV- } \\
\mathbf{n = 4 9 4}\end{array}$ & $p=$ \\
\hline Pangenotypic & & & \\
SOF + RBV & $3(1.8)$ & $3(0.6)^{*}$ & 0.17 \\
GLE/PIB & $34(20.2)$ & $70(14.2)$ & 0.07 \\
SOF/VEL \pm RBV & $41(24.4)$ & $67(13.6)$ & 0.002 \\
\hline Current genotype-specific regimens, $\mathrm{n}(\%)$ & $90(53.6)$ & $354(71.7)$ & \\
Current pangenotypic treatment regimens, $\mathrm{n}(\%)$ & $78(46.4)$ & $140(28.3)$ & $<0.001$ \\
\hline
\end{tabular}

HIV, human immunodeficiency virus; IFN, interferon; PegIFN, pegylated interferon; RBV, ribavirin; SOF, sofosbuvir; SMV, simeprevir; LDV, ledipasvir; OPr, ombitasvir, paritaprevir boosted ritonavir; GZR, grazoprevir; EBR, elbasvir; ASV, asunaprevir; DCV, daclatasvir; GLE, glecaprevir; PIB, pibrentasvir; VEL, velpatasvir. * One patient received a regimen with $\mathrm{DCV}$.

In those with failure of the previous antiviral therapy, the pegylated interferon and ribavirin was the most common option used in both subpopulations. The genotype-specific regimen of OPr + RBV was more commonly used in HCV monoinfected patients, $42.9 \%$ vs. $29.8 \%, p=0.003$. Pangenotypic options were significantly more frequently applied in HIV-positive patients, $46.4 \%$ vs. $28.3 \%, p=0.003$ in HCV monoinfected individuals.

Of the pangenotypic regimens, the combination of SOF/VEL \pm RBV was significantly more common in HCV/HIV-coinfected ( $24.4 \%$ vs. $13.6 \%, p=0.002)$, while the absolute difference in GLE/PIB application was not statistically significant (20.2\% vs. $14.2 \%, p=0.07)$.

\subsection{Efficacy}

The overall sustained virologic response according to ITT analysis was $94.9 \%$, but after exclusion of non-virologic failures, it reached $98 \%$ with no significant difference between $\mathrm{HCV} / \mathrm{HIV}$ coinfected and HCV monoinfected patients, $96.2 \%$ vs. $98.5 \%$ respectively. A significant difference in the SVR rate was found when the comparison was performed according to ITT analysis due to a higher percentage of lost to follow-up patients among $\mathrm{HCV} / \mathrm{HIV}$-coinfected individuals, $91.7 \%$ vs. $96 \%, p=0.04$.

The analysis considering the type o regimen used revealed that genotype-specific regimens resulted in a comparable SVR rate regardless of the HIV status, 97.7\% of HIVcoinfected and $98.3 \%$ of HCV-monoinfected individuals responded to therapy, $p=0.67$ in the PP analysis. In the case of pangenotypic regimens, the HIV-negative patients responded in a higher percentage than HIV-positive, $99.3 \%$ vs. 94.4\%, with the difference on the verge of statistical significance in the PP analysis $(p=0.05)$ and significant in ITT analysis $(p=0.007)($ Table 4$)$.

Table 4. Treatment effectiveness following genotype-specific and pangenotypic regimens according to the HIV status.

\begin{tabular}{cccccc}
\hline & & HIV & HIV & $p$ & $\begin{array}{c}\text { OR } \\
\mathbf{( 9 5 \% C I ) ~}\end{array}$ \\
\hline \multirow{2}{*}{ All } & ITT & $154 / 168(91.7)$ & $474 / 494(96.0)$ & 0.04 & $0.46(0.23-0.94)$ \\
\cline { 2 - 6 } & PP & $154 / 160(96.2)$ & $474 / 481(98.5)$ & 0.10 & $0.38(0.12-1.14)$ \\
\hline \multirow{2}{*}{ Genotype-specific } & ITT & $86 / 90(95.6)$ & $338 / 354(95.5)$ & 1.00 & $1.02(0.33-3.12)$ \\
\cline { 2 - 7 } & PP & $86 / 88(97.7)$ & $338 / 344(98.3)$ & 0.67 & $0.76(0.15-3.85)$ \\
\hline \multirow{2}{*}{ Pangenotypic } & ITT & $68 / 78(87.2)$ & $136 / 140(97.1)$ & 0.007 & $0.20(0.06-0.66)$ \\
\cline { 2 - 6 } & PP & $68 / 72(94.4)$ & $136 / 137(99.3)$ & 0.05 & $0.12(0.01-1.14)$ \\
\hline
\end{tabular}

HIV, human immunodeficiency virus; ITT, intent to treat; PP, per protocol; OR, odds ratio; $\mathrm{CI}$, confidence interval.

In the evaluation carried out in the subpopulations of patients considering the type of therapy, no significant difference in the treatment efficacy with genotype-specific ver- 
sus pangenotypic options was found in either HIV negative or HIV positive individuals (Figure 2).

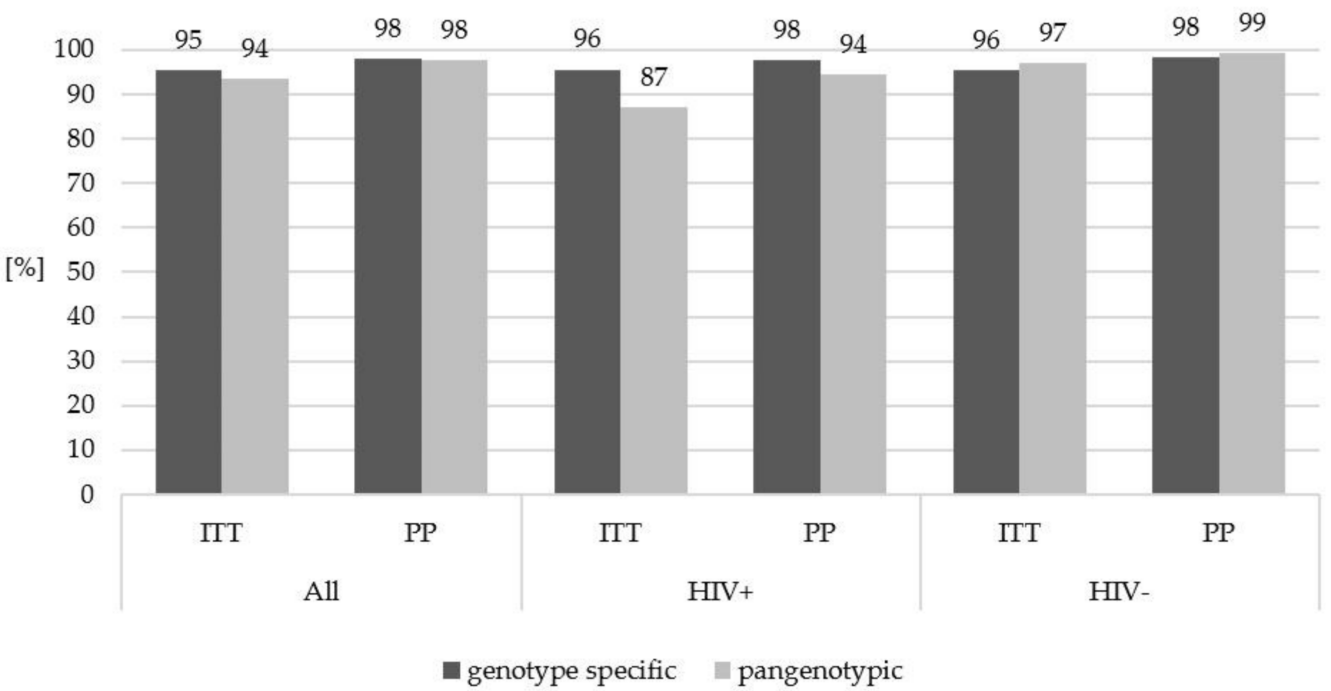

Figure 2. Treatment effectiveness in HIV-positive and HIV-negative patients according to the type of therapeutic regimen used. HIV, human immunodeficiency virus; ITT, intent to treat; PP, per protocol. No statistically significant differences were found between the analyzed groups.

Thirteen virologic non-responders, six HCV / HIV-coinfected and seven HCV monoinfected, were documented (Table 5).

Table 5. Characteristics of 13 virologic failures.

\begin{tabular}{|c|c|c|c|c|c|c|c|c|c|c|}
\hline Patient & Age & HIV+ & $\mathrm{F}, \mathrm{CP}$ & Regimen & $\begin{array}{l}\text { History of } \\
\text { Previous } \\
\text { Therapy }\end{array}$ & $\begin{array}{c}\text { ART } \\
\text { Regimen }\end{array}$ & $\begin{array}{c}\text { Baseline } \\
\text { HCV } \\
\text { RNA IU/mL }\end{array}$ & $\begin{array}{l}\text { Treatment } \\
\text { Course } \\
\text { Completed }\end{array}$ & EOT & Comment \\
\hline Female 1 & 35 & yes & 1 & $\begin{array}{l}\text { GLE/PIB, } \\
8 \text { weeks }\end{array}$ & treatment-naive & $\begin{array}{c}\text { abacavir, } \\
\text { lamivudin, } \\
\text { dolutegravir }\end{array}$ & $2,700,000$ & $\begin{array}{l}\text { according } \\
\text { to schedule }\end{array}$ & TND & \\
\hline Male 1 & 47 & yes & 1 & $\begin{array}{l}\mathrm{OPr}+\mathrm{RBV} \\
12 \text { weeks }\end{array}$ & relapser (PR) & $\begin{array}{l}\text { emtricitabin, } \\
\text { tenofovir } \\
\text { alafenamide }\end{array}$ & $4,538,833$ & $\begin{array}{l}\text { according } \\
\text { to schedule }\end{array}$ & TD & \\
\hline Male 2 & 52 & yes & $4, \mathrm{CP} A$ & $\begin{array}{l}\text { GZR/EBR, } \\
12 \text { weeks }\end{array}$ & treatment-naive & $\begin{array}{c}\text { abacavir, } \\
\text { lamivudin, } \\
\text { dolutegravir }\end{array}$ & $1,890,000$ & $\begin{array}{l}\text { according } \\
\text { to schedule }\end{array}$ & TND & \\
\hline Male 3 & 28 & yes & 1 & $\begin{array}{l}\text { GLE/PIB, } \\
8 \text { weeks }\end{array}$ & treatment-naive & $\begin{array}{l}\text { emtricitabin, } \\
\text { tenofovir } \\
\text { alafenamide, } \\
\text { cobicistat, } \\
\text { elvitegravir }\end{array}$ & 473,000 & $\begin{array}{l}\text { according } \\
\text { to schedule }\end{array}$ & TND & \\
\hline Male 4 & 30 & yes & 2 & $\begin{array}{l}\text { GLE/PIB, } \\
8 \text { weeks }\end{array}$ & treatment-naive & $\begin{array}{l}\text { emtricitabin, } \\
\text { tenofovir } \\
\text { alafenamide, } \\
\text { cobicistat, } \\
\text { elvitegravir }\end{array}$ & 863,000 & $\begin{array}{l}\text { according } \\
\text { to schedule }\end{array}$ & TND & \\
\hline Male 5 & 44 & yes & 1 & $\begin{array}{l}\text { GLE/PIB, } \\
8 \text { weeks }\end{array}$ & treatment-naive & none & 668,519 & $\begin{array}{l}\text { according } \\
\text { to schedule }\end{array}$ & TD & \\
\hline Female 2 & 46 & no & 1 & $\begin{array}{l}\text { SOF/VEL, } \\
12 \text { weeks }\end{array}$ & treatment-naive & na & 542,000 & $\begin{array}{l}\text { according } \\
\text { to schedule }\end{array}$ & $\mathrm{TD}$ & \\
\hline Female 3 & 78 & no & $4, \mathrm{CP} A$ & $\begin{array}{l}\text { OPr+RBV, } \\
12 \text { weeks }\end{array}$ & $\begin{array}{l}\text { null responder } \\
\text { (PR) }\end{array}$ & na & $1,378,790$ & $\begin{array}{l}\text { according } \\
\text { to schedule }\end{array}$ & TND & \\
\hline Female 4 & 25 & no & 1 & $\begin{array}{c}\text { GZR/EBR+RBV, } \\
12 \text { weeks }\end{array}$ & treatment-naive & na & $3,561,961$ & $\begin{array}{l}\text { according } \\
\text { to schedule }\end{array}$ & TND & \\
\hline Female 5 & 79 & no & 2 & $\begin{array}{l}\text { GZR/EBR, } \\
12 \text { weeks }\end{array}$ & treatment-naive & na & 91,900 & $\begin{array}{l}\text { according } \\
\text { to schedule }\end{array}$ & TND & \\
\hline
\end{tabular}


Table 5. Cont.

\begin{tabular}{|c|c|c|c|c|c|c|c|c|c|c|}
\hline Patient & Age & HIV+ & $\mathrm{F}, \mathrm{CP}$ & Regimen & $\begin{array}{l}\text { History of } \\
\text { Previous } \\
\text { Therapy }\end{array}$ & $\begin{array}{c}\text { ART } \\
\text { Regimen }\end{array}$ & $\begin{array}{c}\text { Baseline } \\
\text { HCV } \\
\text { RNA IU/mL }\end{array}$ & $\begin{array}{l}\text { Treatment } \\
\text { Course } \\
\text { Completed }\end{array}$ & EOT & Comment \\
\hline Male 6 & 48 & no & 1 & $\begin{array}{l}\text { OPr+RBV, } \\
12 \text { weeks }\end{array}$ & $\begin{array}{c}\text { relapser } \\
(\mathrm{PR}+\mathrm{SMV})\end{array}$ & na & $3,600,000$ & $\begin{array}{l}\text { according } \\
\text { to schedule }\end{array}$ & TND & $\begin{array}{c}\text { non- } \\
\text { adherence }\end{array}$ \\
\hline Male 7 & 46 & no & 4, CP В & $\begin{array}{l}\text { LDV /SOF, } \\
12 \text { weeks }\end{array}$ & treatment-naive & na & 317,281 & $\begin{array}{l}\text { according } \\
\text { to schedule }\end{array}$ & TND & \\
\hline Male 8 & 25 & no & 1 & $\begin{array}{l}\text { GZR/EBR, } \\
12 \text { weeks }\end{array}$ & $\begin{array}{l}\text { null-responder } \\
\text { (PR) }\end{array}$ & na & $6,320,000$ & $\begin{array}{l}\text { according } \\
\text { to schedule }\end{array}$ & $\mathrm{TD}$ & \\
\hline
\end{tabular}

HIV, human immunodeficiency virus; F, fibrosis; $\mathrm{CP}$, Child-Pugh scale; ART, antiretroviral; HCV RNA, ribonucleic acid of hepatitis $C$ virus; EOT, end of treatment; GLE, glecaprevir; PIB, pibrentasvir; TND, target not detected; OPr, ombitasvir, paritaprevir boosted ritonavir; RBV, ribavirin; PR, Pegylated interferon + Ribavirin; TD, target detected; GZR, grazoprevir; EBR, elbasvir; SOF, sofosbuvir; VEL, velpatasvir; na, not applicable; SMV, simeprevir; LDV, ledipasvir.

Five of them were females, three patients were diagnosed with liver cirrhosis, including two scoring as $\mathrm{A}$ and one as $\mathrm{B}$ on the $\mathrm{CP}$ scale. Four treatment failures were previously treated with IFN-based regimens, three received pegIFN + RBV and one simeprevir + pegIFN + RBV. One of six HIV-coinfected nonresponders was not treated with ART while three were on emtricitabine + tenofovir alafenamide, two of them with cobicistat and elvitegravir and one with darunavir, two remaining HIV positive failures received abacavir + lamivudine and dolutegravir. All patients who did not respond to therapy completed the treatment course according to schedule and nine were negative for $\mathrm{HCV}$ RNA at the end of therapy.

No significant differences in demographic, clinical, and laboratory parameters were found in virologic failures compared to responders (Supplementary Table S1).

Comparative analysis by HIV status showed no statistically significant differences between responders and virologic non-responders in both HCV monoinfection and HIV coinfection groups.

The analysis of the SVR in specific subpopulations regarding gender, BMI, presence of the liver fibrosis F4, and the history of IFN-free therapy is presented in Table 6.

Table 6. Treatment effectiveness in subpopulations.

\begin{tabular}{ccc}
\hline & SVR ITT & SVR PP \\
\hline Females, $\mathrm{n}=263$ & $252 / 263(95.8)$ & $252 / 257(98.1)$ \\
\hline Males, $\mathrm{n}=399$ & $376 / 399(94.2)$ & $376 / 384(97.9)$ \\
\hline IFN-free failure, $\mathrm{n}=11$ & $10 / 11(91)$ & $10 / 10(100)$ \\
\hline BMI $>$ 30, $\mathrm{n}=108$ & $101 / 108(93.5)$ & $101 / 103(98.1)$ \\
\hline Fibrosis F4, $\mathrm{n}=105$ & $98 / 105(93.3)$ & $98 / 101(97)$ \\
\hline SVR, sustained virologic response; ITT, intent to treat; PP, per protocol; IFN, interferon; BMI, body mass index.
\end{tabular}

The majority of patients completed therapy according to schedule $(95.8 \%)$ no impact of HIV-coinfection was observed (Supplementary Table S2).

\subsection{Safety}

Treatment modification in the form of reduction or discontinuation of the RBV was documented in seven patients. In one HIV-negative individual therapy was discontinued due to liver decompensation. At least one $\mathrm{AE}$ was documented in $23 \%$ of patients, the most common were weakness/fatigue and anemia noticed significantly more frequently in HCV monoinfected patients $(0.01 \%$ vs. $5.1 \%, p=0.02)$. One death due to non-liver cancer in monoinfected population and three serious $\mathrm{AE}$ were documented during the treatment course and follow-up period. 


\section{Discussion}

The overlapping transmission routes resulted in a high prevalence of $\mathrm{HCV} / \mathrm{HIV}$ coinfection. It is estimated that approximately $25 \%$ of patients living with HIV are coinfected with HCV worldwide. However, the prevalence rates vary significantly across subpopulations and geographic regions [21,22].

The introduction of the DAA options meant that this population is now treated equally to those infected with HCV only. To the best of our knowledge, the analyzed cohort is the most numerous population of HCV GT4 patients with $\mathrm{HCV} / \mathrm{HIV}$ coinfection who have undergone DAA therapy, including pangenotypic regimens, to date. It should be emphasized that we have not confirmed the progression of hepatic fibrosis and more severe liver disease in this coinfected population, as reported by other researchers [23]. On the contrary, the rate of cirrhotic patients among GT4 infected HIV-positive population was significantly lower compared to that with HCV GT4 monoinfection, and any coinfected patient with liver cirrhosis presented hepatic decompensation at baseline. Furthermore, none of the coinfected individuals in the analyzed cohort had a history of hepatocellular carcinoma or liver transplantation.

A significantly higher percentage of treatment-naïve patients in the HIV-positive group in the current study confirms that in the era of DAA they gained wider access to antiviral therapy compared to the previous period, which is supported by the data from the literature [11].

The current study demonstrated the overall high efficacy of the DAA treatment in $\mathrm{HCV} / \mathrm{HIV}$ coinfected patients, not different from the effectiveness obtained in the group of those with HCV GT4 monoinfection. Our data on the comparable SVR rate regardless of the HIV status support results from numerous clinical trials with the usage of different DAA regimens in HIV positive GT4 infected patients [14-18,24]. In groups of 5 to $30 \mathrm{HCV}$ GT4/HIV-coinfected participants of C-EDGE CO-INFECTION (GZR/EBR), ASTRAL-5 (SOF/VEL), TURQOISE-I (OPr \pm DSV \pm RBV), EXPEDITION-2 (GLE/PIB), ION-4 (SOF/LDV), and HEPNED-001 studies, effectiveness of 93-100\% following therapy with different DAA regimens was reported [14-18,24].

Similar efficacy was documented in real-world studies of HCV / HIV coinfection conducted by Machado et al., Piekarska et al., Bischoff et al., Navarro et al., and Minosse et al., but only some of them included a larger group of GT4 infected patients (33-77 participants) [25-29]. In the abovementioned real-world analyses, patients were treated predominantly with genotype-specific regimens, only a few patients received the first available pangenotypic option of $\mathrm{SOF} \pm \mathrm{DCV} \pm \mathrm{RBV}$.

Even a real-world study of the largest cohort of HIV-positive patients with HCV GT4 infection to date conducted by Berenguer et al. did not evaluate the new pangenotypic regimens [30]. Of the 530 such patients accounting for $22.4 \%$ of all analyzed $\mathrm{HCV} / \mathrm{HIV}$ coinfected Spanish population, 34\% were diagnosed with liver cirrhosis. They were treated almost exclusively with two genotype-specific regimens, SOF/LDV and OPr and the cure rates in modified ITT analysis were $95.5 \%$ and $94.7 \%$ in patients without cirrhosis, and $93.9 \%$ and $100 \%$ in those with cirrhosis, respectively. Twenty patients with decompensated liver cirrhosis receiving SOF/LDV achieved a significantly lower SVR of $80 \%$. One GT4 HCV/HIV-coinfected patient was treated with SOF + DCV and another one with SOF + SMV, both achieved an SVR [30].

Another real-world study was conducted by Sousa et al. in Spain on just one DAA regimen of $\mathrm{OPr} \pm \mathrm{RBV}$ with or without dasabuvir covering a group of $2408 \mathrm{HCV}$ patients of whom 386 patients were HIV coinfected showed that infection with GT4 was associated with non-response to treatment in the multivariate analysis [31].

The current study documented an SVR rate of $98 \%$ for genotype-specific regimens regardless of the HIV status, while the effectiveness of the pangenotypic options (almost exclusively GLE/PIB and SOF/VEL combinations) was lower among GT4 HCV/HIV coinfected individuals and did not reach $95 \%$ as compared to $99 \%$ in HCV monoinfected subpopulation. Due to the lack of real-world studies evaluating new pangenotypic options 
in HIV coinfected GT4 HCV patients, clinical trials EXPEDITION-2 and ASTRAL-5 remain the only point of reference [15,17]. In both studies, the SVR was achieved by all HIV coinfected GT4 participants, but the small number of such patients should be highlighted, 17 and 5 individuals, respectively. One of the possible explanations for this discrepancy could be a relatively high rate of non-virologic nonresponders among HIV coinfected patients treated with pangenotypic regimens in our analysis, $7 \%$ compared to $2 \%$ in the genotypespecific arm. It can be assumed that the negative results in these lost-to-follow-up patients could affect the final effectiveness. All four virologic nonresponders to pangenotypic regimens were treated with the combination of GLE/PIB which is an unexpected finding requiring further analysis in the larger population. No possible drug-drug interactions between the ART and DAAs were expected, all of them were on the emtricitabine/tenofovir alafenamide, two received also cobicistat and elvitegravir. They were treated according to the label of GLE/PIB for 8 weeks, all of them were treatment-naïve and diagnosed with liver fibrosis F1-F2.

The likely reason for the higher efficacy of genotype specific therapy in HIV coinfected GT4-infected patients is that it had been used before when most patients waited in line to access new interferon-free treatment options, and thus showed greater cure determination and adherence. Unfortunately, our study did not assess adherence, so we cannot confirm this hypothesis. Moreover, $\mathrm{HCV}$ reinfection during the post-treatment follow-up period in these patients cannot be ruled out since such a phenomenon was demonstrated previously $[32,33]$.

There were no safety issues during DAA therapy in GT4 HCV / HIV coinfected patients, no hepatic decompensation and no death were reported and only one serious AE not related to DAA treatment was documented, the frequency of AEs was comparable to HCV monoinfected patients. It should be highlighted that the selection of the therapeutic regimen was made upon the baseline evaluation of the ART to avoid the possible drug-drug interactions which could result in worse tolerability. Our findings on the favorable safety profile are in accordance with numerous clinical trials and real-world studies conducted in patients with HIV / HCV coinfection [15,17,26,30,34,35].

There are some limitations of the current study that should be considered when interpreting the results. One of them is observational design and retrospective data collecting with possible DAA selection bias, which is a result of real-world investigator decision study design. Some potentially useful data are missing, including drug monitoring and objective adherence records; also, the adverse events may be underreported in real-world settings. Another limitation is the relatively small sample sizes of HIV coinfected patients in the context of single treatment DAA regimens, especially DAA-failures among whom only three had liver cirrhosis and four previous anti-HCV failure preventing the performance of multivariate analysis. Finally, no baseline resistance mutation testing was performed.

However, the main strength of the current analysis is the largest number of $\mathrm{HIV} / \mathrm{HCV}$ GT4 coinfected patients treated with DAA using both genotype-specific and pangenotypic regimens, to date. This multicenter analysis covers a heterogeneous population, representative of the real-world study. We use a population of HCV GT4 monoinfected patients as a comparator group.

\section{Conclusions}

We confirmed the high effectiveness and good safety profile of the DAA therapy in the population of HCV GT4 infected patients with HIV coinfection supporting the current recommendations to treat $\mathrm{HCV} / \mathrm{HIV}$ coinfected patients with the same options as those with HCV monoinfection. The comparative analysis considering the type of regimen used documented the lower SVR achieved by those treated with pangenotypic options. Further studies are needed to allow the accumulation of non-responders to determine the actual outcomes of HCV GT4 treatment with pangenotypic regimens in HIV co-infected. 
Supplementary Materials: The following supporting information can be downloaded at: https: / / www.mdpi.com/article/10.3390/jcm11020389/s1, Table S1: Virologic nonresponders versus responders among GT4-infected patients; Table S2: Safety of antiviral treatment in GT4-infected patients.

Author Contributions: Conceptualization-D.Z.-M. and R.F.; Methodology-D.Z.-M. and R.F.; Formal analysis-D.Z.-M. and J.J.; Investigation-D.Z.-M.; Validation-R.F.; Writing—original draft preparation-D.Z.-M.; Writing—review and editing-R.F.; Supervision-R.F.; Project administrationR.F.; Data collection-all authors. All authors have read and agreed to the published version of the manuscript.

Funding: This research received no external funding.

Institutional Review Board Statement: This observational study was conducted in a real-world setting with approved drugs. Patients were not exposed to any experimental interventions nor did the study intervene with the clinical management of the patient. The study only collected information from patient medical records. The analysis included routine examinations and tests performed in patients treated within the therapeutic program of the National Health Fund. The data were originally collected to assess treatment efficacy and safety in individual patients, not for scientific purposes. Hence, the treating physicians did not obtain approval from the ethics committee. According to local law (Pharmaceutical Law of 6 September 2001, art. 37al), non-interventional studies do not require ethics committee approval.

Informed Consent Statement: Patient consent was waived due to the retrospective design of the study.

Data Availability Statement: Data supporting reported results can be provided upon request from the corresponding author.

Conflicts of Interest: The authors declare no conflict of interest.

\section{References}

1. Blach, S.; Zeuzem, S.; Manns, M.; Altraif, I.; Duberg, A.-S.; Muljono, D.H.; Waked, I.; Alavian, S.M.; Lee, M.-H.; Negro, F.; et al. Global Prevalence and Genotype Distribution of Hepatitis C Virus Infection in 2015: A Modelling Study. Lancet Gastroenterol. Hepatol. 2017, 2, 161-176. [CrossRef]

2. Fernández-Arcás, N.; López-Siles, J.; Trapero, S.; Ferraro, A.; Ibáñez, A.; Orihuela, F.; Maldonado, J.; Alonso, A. High Prevalence of Hepatitis C Virus Subtypes 4c and 4d in Malaga (Spain): Phylogenetic and Epidemiological Analyses. J. Med. Virol. 2006, 78, 1429-1435. [CrossRef] [PubMed]

3. Kamal, S.M.; Nasser, I.A. Hepatitis C Genotype 4: What We Know and What We Don't yet Know. Hepatology 2008, 47, 1371-1383. [CrossRef]

4. Franco, S.; Tural, C.; Clotet, B.; Martínez, M.A. Complete Nucleotide Sequence of Genotype 4 Hepatitis C Viruses Isolated from Patients Co-Infected with Human Immunodeficiency Virus Type 1. Virus Res. 2007, 123, 161-169. [CrossRef] [PubMed]

5. De Bruijne, J.; Schinkel, J.; Prins, M.; Koekkoek, S.M.; Aronson, S.J.; van Ballegooijen, M.W.; Reesink, H.W.; Molenkamp, R.; van de Laar, T.J.W. Emergence of Hepatitis C Virus Genotype 4: Phylogenetic Analysis Reveals Three Distinct Epidemiological Profiles. J. Clin. Microbiol. 2009, 47, 3832-3838. [CrossRef] [PubMed]

6. Abdel-Ghaffar, T.Y.; Sira, M.M.; El Naghi, S. Hepatitis C Genotype 4: The Past, Present, and Future. World J. Hepatol. 2015, 7, 2792-2810. [CrossRef]

7. Jacobson, I.; Zeuzem, S.; Flisiak, R.; Knysz, B.; Lueth, S.; Zarebska-Michaluk, D.; Janczewska, E.; Ferenci, P.; Diago, M.; Zignego, A.L.; et al. Daclatasvir vs Telaprevir plus Peginterferon Alfa/Ribavirin for Hepatitis C Virus Genotype 1. World J. Gastroenterol. 2016, 22, 3418-3431. [CrossRef]

8. Moreno, C.; Hezode, C.; Marcellin, P.; Bourgeois, S.; Francque, S.; Samuel, D.; Zoulim, F.; Grange, J.-D.; Shukla, U.; Lenz, O.; et al. Efficacy and Safety of Simeprevir with PegIFN/Ribavirin in Naïve or Experienced Patients Infected with Chronic HCV Genotype 4. J. Hepatol. 2015, 62, 1047-1055. [CrossRef]

9. Lawitz, E.; Mangia, A.; Wyles, D.; Rodriguez-Torres, M.; Hassanein, T.; Gordon, S.C.; Schultz, M.; Davis, M.N.; Kayali, Z.; Reddy, K.R.; et al. Sofosbuvir for Previously Untreated Chronic Hepatitis C Infection. N. Engl. J. Med. 2013, 368, 1878-1887. [CrossRef]

10. Hézode, C.; Alric, L.; Brown, A.; Hassanein, T.; Rizzetto, M.; Buti, M.; Bourlière, M.; Thabut, D.; Molina, E.; Rustgi, V.; et al. Randomized Controlled Trial of the NS5A Inhibitor Daclatasvir plus Pegylated Interferon and Ribavirin for HCV Genotype-4 (COMMAND-4). Antivir. Ther. 2015, 21, 195-205. [CrossRef]

11. Sulkowski, M.S.; Benhamou, Y. Therapeutic Issues in HIV/HCV-Coinfected Patients. J. Viral. Hepat. 2007, 14, 371-386. [CrossRef]

12. Flisiak, R.; Pogorzelska, J.; Berak, H.; Horban, A.; Orłowska, I.; Simon, K.; Tuchendler, E.; Madej, G.; Piekarska, A.; Jabłkowski, M.; et al. Efficacy of HCV Treatment in Poland at the Turn of the Interferon Era-The EpiTer Study. Clin. Exp. Hepatol. 2016, 2, 138-143. [CrossRef] 
13. Di Biagio, A.; Taramasso, L.; Cenderello, G. Treatment of Hepatitis C Virus Genotype 4 in the DAA Era. Virol. J. 2018, 15, 180. [CrossRef]

14. Rockstroh, J.K.; Nelson, M.; Katlama, C.; Lalezari, J.; Mallolas, J.; Bloch, M.; Matthews, G.V.; Saag, M.S.; Zamor, P.J.; Orkin, C.; et al. Efficacy and Safety of Grazoprevir (MK-5172) and Elbasvir (MK-8742) in Patients with Hepatitis C Virus and HIV Co-Infection (C-EDGE CO-INFECTION): A Non-Randomised, Open-Label Trial. Lancet HIV 2015, 2, e319-e327. [CrossRef]

15. Wyles, D.; Bräu, N.; Kottilil, S.; Daar, E.S.; Ruane, P.; Workowski, K.; Luetkemeyer, A.; Adeyemi, O.; Kim, A.Y.; Doehle, B.; et al. Sofosbuvir and Velpatasvir for the Treatment of Hepatitis C Virus in Patients Coinfected With Human Immunodeficiency Virus Type 1: An Open-Label, Phase 3 Study. Clin. Infect. Dis. 2017, 65, 6-12. [CrossRef]

16. Rockstroh, J.K.; Orkin, C.; Viani, R.M.; Wyles, D.; Luetkemeyer, A.F.; Lazzarin, A.; Soto-Malave, R.; Nelson, M.R.; Bhagani, S.R.; Klinker, H.H.F.; et al. Safety and Efficacy of Ombitasvir, Paritaprevir With Ritonavir \pm Dasabuvir With or Without Ribavirin in Patients With Human Immunodeficiency Virus-1 and Hepatitis C Virus Genotype 1 or Genotype 4 Coinfection: TURQUOISE-I Part 2. Open Forum Infect. Dis. 2017, 4, ofx154. [CrossRef]

17. Rockstroh, J.K.; Lacombe, K.; Viani, R.M.; Orkin, C.; Wyles, D.; Luetkemeyer, A.F.; Soto-Malave, R.; Flisiak, R.; Bhagani, S.; Sherman, K.E.; et al. Efficacy and Safety of Glecaprevir/Pibrentasvir in Patients Coinfected With Hepatitis C Virus and Human Immunodeficiency Virus Type 1: The EXPEDITION-2 Study. Clin. Infect. Dis. 2018, 67, 1010-1017. [CrossRef]

18. Naggie, S.; Cooper, C.; Saag, M.; Workowski, K.; Ruane, P.; Towner, W.J.; Marks, K.; Luetkemeyer, A.; Baden, R.P.; Sax, P.E.; et al Ledipasvir and Sofosbuvir for HCV in Patients Coinfected with HIV-1. N. Engl. J. Med. 2015, 373, 705-713. [CrossRef]

19. Halota, W.; Flisiak, R.; Juszczyk, J.; Małkowski, P.; Pawłowska, M.; Simon, K.; Tomasiewicz, K. Recommendations of the Polish Group of Experts for HCV for the Treatment of Hepatitis C in 2020. Clin. Exp. Hepatol. 2020, 6, 163-169. [CrossRef]

20. European Association for the Study of the Liver. EASL Recommendations on Treatment of Hepatitis C 2018. J. Hepatol. 2018, 69, 461-511. [CrossRef]

21. Soriano, V.; Vispo, E.; Labarga, P.; Medrano, J.; Barreiro, P. Viral Hepatitis and HIV Co-Infection. Antivir. Res. 2010, 85, 303-315. [CrossRef]

22. Grzeszczuk, A.; Wandalowicz, A.D.; Jaroszewicz, J.; Flisiak, R. Prevalence and Risk Factors of HCV /HIV Co-Infection and HCV Genotype Distribution in North-Eastern Poland. Hepat. Mon. 2015, 15, e27740. [CrossRef]

23. Kirk, G.D.; Mehta, S.H.; Astemborski, J.; Galai, N.; Washington, J.; Higgins, Y.; Balagopal, A.; Thomas, D.L. HIV, Age, and the Severity of Hepatitis C Virus-Related Liver Disease. Ann. Intern. Med. 2013, 158, 658-666. [CrossRef]

24. Boerekamps, A.; Vanwolleghem, T.; van der Valk, M.; van den Berk, G.E.; van Kasteren, M.; Posthouwer, D.; Dofferhoff, A.S.M.; van Hoek, B.; Ramsoekh, D.; Koopsen, J.; et al. 8 Weeks of Sofosbuvir/Ledipasvir Is Effective in DAA-Naive Non-Cirrhotic HCV Genotype 4 Infected Patients (HEPNED-001 Study). J. Hepatol. 2019, 70, 554-557. [CrossRef]

25. Machado, S.M.; Vigani, A.G.; Leite, A.G.; Diaz, A.C.M.; Ferreira, P.R.A.; Carnaúba-Júnior, D.; Tenore, S.B.; Brandão-Mello, C.E.; Gonzalez, M.P.; Siroma, F.; et al. Effectiveness of Direct-Acting Antivirals for Hepatitis C Virus Infection in Hepatitis C/HIV Coinfected Individuals. Medicine 2020, 99, e21270. [CrossRef]

26. Piekarska, A.; Jabłonowska, E.; Garlicki, A.; Sitko, M.; Mazur, W.; Jaroszewicz, J.; Czauz-Andrzejuk, A.; Buczyńska, I.; Simon, K.; Lorenc, B.; et al. Real Life Results of Direct Acting Antiviral Therapy for HCV Infection in HIV-HCV-Coinfected Patients: Epi-Ter2 Study. AIDS Care 2020, 32, 762-769. [CrossRef]

27. Bischoff, J.; Mauss, S.; Cordes, C.; Lutz, T.; Scholten, S.; Moll, A.; Jäger, H.; Cornberg, M.; Manns, M.; Baumgarten, A.; et al. Rates of Sustained Virological Response 12 Weeks after the Scheduled End of Direct-Acting Antiviral (DAA)-Based Hepatitis C Virus (HCV) Therapy from the National German HCV Registry: Does HIV Coinfection Impair the Response to DAA Combination Therapy? HIV Med. 2018, 19, 299-307. [CrossRef] [PubMed]

28. Navarro, J.; Laguno, M.; Vilchez, H.H.; Guardiola, J.M.; Carrion, J.A.; Force, L.; Cairó, M.; Cifuentes, C.; Vilaró, J.; Cucurull, J.; et al. Efficacy and Safety of Direct Antiviral Agents in a Cohort of Cirrhotic HCV/HIV-Coinfected Patients. J. Antimicrob. Chemother. 2017, 72, 2850-2856. [CrossRef]

29. Minosse, C.; Selleri, M.; Giombini, E.; Bartolini, B.; Capobianchi, M.R.; Cerilli, S.; Loiacono, L.; Taibi, C.; D'Offizi, G.; McPhee, F.; et al. Clinical and Virological Properties of Hepatitis C Virus Genotype 4 Infection in Patients Treated with Different Direct-Acting Antiviral Agents. Infect. Drug Resist. 2018, 11, 2117-2127. [CrossRef] [PubMed]

30. Berenguer, J.; Gil-Martin, Á.; Jarrin, I.; Moreno, A.; Dominguez, L.; Montes, M.; Aldámiz-Echevarría, T.; Téllez, M.J.; Santos, I.; Benitez, L.; et al. All-oral Direct-acting Antiviral Therapy against Hepatitis C Virus (HCV) in Human Immunodeficiency Virus/HCV-Coinfected Subjects in Real-world Practice: Madrid Coinfection Registry Findings. Hepatology 2018, 68, 32-47. [CrossRef] [PubMed]

31. Manuel Sousa, J.; Vergara, M.; Pulido, F.; Sánchez Antolín, G.; Hijona, L.; Carnicer, F.; Rincón, D.; Salmerón, J.; Mateos-Muñoz, B.; Jou, A.; et al. Real-World Evidence of the Effectiveness of Ombitasvir-Paritaprevir $/ \mathrm{r} \pm$ Dasabuvir \pm Ribavirin in Patients Monoinfected with Chronic Hepatitis C or Coinfected with Human Immunodeficiency Virus-1 in Spain. PLoS ONE 2019, 14, e0225061. [CrossRef]

32. Adu, P.A.; Rossi, C.; Binka, M.; Wong, S.; Wilton, J.; Wong, J.; Butt, Z.A.; Bartlett, S.; Jeong, D.; Pearce, M.; et al. HCV Reinfection Rates after Cure or Spontaneous Clearance among HIV-Infected and Uninfected Men Who Have Sex with Men. Liver Int. 2021, 41, 482-493. [CrossRef] 
33. Islam, N.; Krajden, M.; Shoveller, J.; Gustafson, P.; Gilbert, M.; Buxton, J.A.; Wong, J.; Tyndall, M.W.; Janjua, N.Z. Incidence, Risk Factors, and Prevention of Hepatitis C Reinfection: A Population-Based Cohort Study. Lancet Gastroenterol. Hepatol. 2017, 2, 200-210. [CrossRef]

34. Zarębska-Michaluk, D.; Jaroszewicz, J.; Buczyńska, I.; Simon, K.; Lorenc, B.; Tudrujek-Zdunek, M.; Tomasiewicz, K.; Sitko, M.; Garlicki, A.; Janczewska, E.; et al. Real-World Experience with Grazoprevir/Elbasvir in the Treatment of Previously "Difficult to Treat" Patients Infected with Hepatitis C Virus Genotype 1 and 4. J. Gastroenterol. Hepatol. 2020, 35, 1238-1246. [CrossRef]

35. Milazzo, L.; Lai, A.; Calvi, E.; Ronzi, P.; Micheli, V.; Binda, F.; Ridolfo, A.L.; Gervasoni, C.; Galli, M.; Antinori, S.; et al. DirectActing Antivirals in Hepatitis C Virus (HCV)-Infected and HCV/HIV-Coinfected Patients: Real-Life Safety and Efficacy. HIV Med. 2017, 18, 284-291. [CrossRef] 